\title{
Adherence to the Dietary Approaches to Stop Hypertension-style diet in relation to glioma: a case-control study
}

\author{
Sanaz Benisi-Kohansal ${ }^{1,2}$, Mehdi Shayanfar ${ }^{3}$, Minoo Mohammad-Shirazi ${ }^{3}$, Hadi Tabibi ${ }^{3}$, Giuve Sharifi ${ }^{3}$, \\ Parvane Saneei ${ }^{1,2,4}$ and Ahmad Esmaillzadeh ${ }^{1,2,5 *}$ \\ ${ }^{1}$ Food Security Research Center, Isfahan University of Medical Sciences, PO Box 81745-151, Isfahan, Iran \\ ${ }^{2}$ Department of Community Nutrition, School of Nutrition and Food Science, Isfahan University of Medical Sciences, PO Box \\ 81745-151, Isfahan, Iran \\ ${ }^{3}$ Department of Clinical Nutrition and Dietetics, National Nutrition and Food Technology Research Institute, Shabid Beheshti \\ University of Medical Sciences, PO Box 40470, Tebran, Iran \\ ${ }^{4}$ Students' Research Committee, Isfahan University of Medical Sciences, PO Box 81745-151, Isfahan, Iran \\ ${ }^{5}$ Department of Community Nutrition, School of Nutritional Sciences and Dietetics, Tebran University of Medical Sciences, \\ PO Box 40450, Tehran, Iran
}

(Submitted 21 August 2015 - Final revision received 23 November 2015 - Accepted 4 December 2015 - First published online 9 February 2016)

\section{Abstract}

Data on the association of adherence to the Dietary Approaches to Stop Hypertension (DASH)-style and glioma are scarce. We aimed to examine the association between adherence to the DASH-style diet and glioma in Iranian adults. In this study, 128 pathologically confirmed cases of glioma were recruited from hospitals and 256 age- and sex-matched controls were enrolled from other wards of the hospital. Dietary intakes were assessed using a 126-item validated FFQ. Adherence to the DASH-style diet was followed considering the healthy and non-healthy foods emphasised in the DASH dietary pattern. After controlling for potential confounders, individuals with the greatest adherence to the DASH diet were $72 \%$ less likely to have glioma compared with those with the lowest adherence (OR $0 \cdot 28 ; 95 \%$ CI $0 \cdot 13,0 \cdot 57$ ). Individuals with the highest consumption of fruits had lower odds for having glioma compared with those with the lowest intake (OR 0.31; 95\% CI 0.14, 0.68). A protective association was also observed between consumption of legumes and nuts and risk of glioma (OR $0 \cdot 23 ; 95 \% \mathrm{CI} 0 \cdot 10,0 \cdot 53)$. We found a significant positive association between red and processed meat (OR 2.60; 95\% CI 1.16, 5.81) and salt intakes (OR 2.87; 95\% CI 1.30, 6.34) and risk of glioma, after taking all potential confounders into account. Adherence to the DASH-style dietary pattern was inversely associated with glioma. In addition, some components of the DASH diet, including red meats and salt intakes, were positively associated with glioma. Consumption of nuts and legumes as well as fruits was inversely associated with glioma. Prospective cohort studies are required to confirm our findings.

\section{Key words: Diet: Glioma: Nutrients: Brain tumour: Dietary Approaches to Stop Hypertension}

Gliomas are the most common type of brain tumours in adults, accounting for $77 \%$ of malignant cases $^{(1)}$. The standardised mortality rates from brain tumours in Iran are 2.92/100 000 in men and 2.46 per 100000 in women ${ }^{(2)}$. Although the incidence of this type of cancer is low, given the high mortality rate of this type of cancer, it is important to identify factors involved in the occurrence.

Diet has long been suggested as a contributing factor to cancer incidence. Dietary determinants of brain tumours have also been widely investigated ${ }^{(3,4)}$. Earlier studies have shown a positive association between dietary intakes of processed meats ${ }^{(5)}$ and fried foods with glioma ${ }^{(6)}$; however, high consumption of fruits and vegetables ${ }^{(7,8)}$ as well as fresh fish and poultry $^{(8)}$ has been linked with a lower risk in adults. Previous studies on diet-glioma associations, mostly of case-control design, have more often than not been focused on nutrients or foods and little attention has been given to dietary patterns ${ }^{(9)}$. Nutritional epidemiologists have suggested using the overall dietary pattern approach, rather than individual nutrients or foods, to find diet-disease relations ${ }^{(10)}$. Such directions would allow reducing the co-linearity problem that might occur when assessing foods and nutrients ${ }^{(10)}$.

The Dietary Approaches to Stop Hypertension (DASH)-style diet is a healthy eating pattern rich in fruits, vegetables, low-fat diary and plant proteins from nuts and legumes and restricted amounts of red meat, sweets and sugar-sweetened beverages $^{(11)}$. A recent meta-analysis has shown the efficacy of this dietary pattern in lowering blood pressure in hypertensive and normotensive individuals ${ }^{(12,13)}$; however, its beneficial effects on other metabolic conditions such as obesity ${ }^{(14)}$, the

Abbreviation: DASH, Dietary Approaches to Stop Hypertension.

* Corresponding author: A. Esmaillzadeh, fax +98 31 36681378, email Esmaillzadeh@hlth.mui.ac.ir 
metabolic syndrome ${ }^{(15)}$ and gestational diabetes ${ }^{(16)}$ have been reported in cross-sectional studies or clinical trials. The effects of this pattern on cancers have been less investigated ${ }^{(17)}$. The favourable associations of fruits and vegetables, as components of the DASH-style diet, with some types of cancers including glioma have been indicated earlier ${ }^{(7,8)}$. Therefore, we hypothesised that adherence to the DASH-style diet might have potential benefits for glioma.

To the best of our knowledge, no earlier study has examined the association between the DASH diet and glioma. In addition, adherence to healthy eating patterns such as the DASH-style diet depends on the food culture of each geographical area, and there are limited data on the adherence to the DASH eating pattern among Iranian adults. It is also worth mentioning that most studies on diet-glioma have been carried out in Western countries, where the dietary intakes are markedly different from Middle Eastern countries ${ }^{(18)}$. People in the Middle East consume large portion sizes containing high amounts of refined grains (white rice and bread) and hydrogenated fats and acquire greater percentage of energy from carbohydrates ${ }^{(18)}$. Therefore, assessing the association between dietary patterns and glioma in a Middle Eastern country might further move the current knowledge forward. Given the inverse relationship between some components of the DASH-style diet and brain tumours in previous studies $^{(7,8)}$, it seems that the whole DASH-style eating pattern might be associated with glioma. This study was, therefore, carried out to investigate the association between the DASH-style diet and glioma in the framework of a case-control study in Iran.

\section{Methods \\ Participants}

This hospital-based case-control study was carried out in Tehran, Iran, between November 2009 and September 2011. Participants, both cases and controls, were selected using convenience-sampling method based on inclusion criteria from the hospitals affiliated to Shahid Beheshti University of Medical Sciences. On the basis of earlier evidence in the country, we hypothesised that approximately $60 \%$ of the Iranian population consume fruit and vegetables lower than the recommended levels ${ }^{(19)}$. We further hypothesised that low fruit and vegetable intakes would double the risk of glioma ${ }^{(4)}$. With $80 \%$ power, type I error of 0.05 and desired CI of 0.95 , the minimum required sample size was calculated to be 115 cases and 230 healthy control subjects. However, we recruited 128 cases and 256 controls from the hospitals. Case participants were individuals with pathologically confirmed glioma (ICD-O-2 morphology codes 9380-9481) during the previous month who had been referred to the Department of Neurosurgery of the hospitals affiliated to Shahid Beheshti University of Medical Sciences. In other words, all patients with glioma were newly diagnosed cases, where a maximum of 1 month had elapsed since the diagnosis of glioma. Cases had to be aged between 20 and 75 years to be included in the study. Individuals with a history of any type of pathologically confirmed cancers (except glioma) and those with a history of chemotherapy or radiotherapy (due to cancer) were not included in the study. Control participants were healthy individuals aged between 20 and 75 years who were hospitalised in other wards (orthopaedic wards or surgery) of the same hospital or were outpatients referring to the same clinic affiliated to Shahid Beheshti University of Medical Sciences. Cases and controls were matched in terms of age $( \pm 5)$ and sex. As we assessed the long-term usual dietary intakes of study participants, the short-term changes in dietary intakes were not considered. All cases and controls provided their written informed consent. The study was ethically approved by the Research Council of Food Security Research Center, Isfahan University of Medical Sciences, Isfahan, Iran.

\section{Dietary assessment}

Usual dietary intakes of the study participants during the preceding year (during the year before the diagnosis of glioma in the case group and during the year before the interview in the control group) were examined using a Willett-format-validated 126-item semi-quantitative $\mathrm{FFQ}^{(20)}$. The FFQ consisted of 126 food items with standard portion sizes commonly consumed by this population. Trained interviewers, who were experienced in completing such questionnaires, administered the FFQ through face-to-face interviews. Interviews with control and case participants were conducted in the presence of individuals who were involved in the preparation and cooking of foods. Participants were asked to indicate their usual consumption frequency of a given serving of food in the preceding year on a daily, weekly or monthly basis. All reported consumption frequencies were converted to grams per day using household measures ${ }^{(21)}$. Subsequently, daily intakes of energy and nutrients were determined using the US Department of Agriculture food composition database ${ }^{(22)}$ that was modified for Iranian foods.

A dietitian who had received the required training on anthropometric measurement techniques and in completing the FFQ and physical activity questionnaire carried out the interviews. The interviewer was totally unaware of the research hypotheses, but she was aware of the participants' condition (in terms of having a disease).

A previous year-long validation study of this FFQ revealed good correlations between dietary intakes assessed by FFQ and those obtained from the $24 \mathrm{~d}$ (two recalls in each month of a year) of 24-h dietary recalls ${ }^{(20)}$. The reliability of the FFQ was assessed by comparing nutrient intakes obtained from the FFQ on two occasions 1-year apart. The correlation coefficients for the reliability of the FFQ for dietary vitamin $\mathrm{E}, \boldsymbol{\beta}$-carotene and vitamin $\mathrm{C}$ were $0.78,0.84$ and 0.83 , respectively. The energy-adjusted correlation coefficients between the dietary intakes obtained from the FFQ and those from the multiple 24-h dietary recalls were 0.65 for vitamin $\mathrm{E}, 0.68$ for $\beta$-carotene and 0.65 for vitamin $\mathrm{C}$. Overall, these data indicated that the FFQ provides reasonably valid measures of the average long-term dietary intakes ${ }^{(20)}$.

\section{Assessment of adherence to the Dietary Approaches to Stop Hypertension-style diet}

We constructed the DASH score based on foods and nutrients emphasised or minimised in the DASH diet, focusing on eight components: high intake of fruits, vegetables, nuts and legumes, 
low-fat dairy products and whole-grains and low intake of salt, sweetened beverages, and red and processed meats ${ }^{(23)}$. We calculated a DASH score for each study participant. First we obtained energy-adjusted amounts of components of the DASH-style diet through residual method ${ }^{(24)}$. Subsequently, quintile cut-off points for each component of the DASH-style diet were obtained in control subjects to avoid the probable bias that might arise from the changes in dietary intakes in case participants. All participants, cases and controls, were then categorised in terms of dietary intakes of the DASH-style components to quintiles based on these cut-off points. For fruits, vegetables, nuts and legumes, low-fat dairy products and whole-grains, those in the first quintile were given the score of 1 and those in the highest quintile were given the score of 5 . Other quintiles (2, 3 and 4) for these dietary intakes were given the corresponding scores. For salt, red and processed meats and sweetened beverages, the lowest quintile was given a score of 5 and the highest quintile was given the score of 1 . Those in quintiles 4,3 and 2 for these dietary intakes were given the scores of 2, 3 and 4, respectively. These scores were then summed up to construct the overall DASH score that ranged from 8 to 40 . Individuals with the highest DASH score were more likely to follow the DASH diet.

\section{Assessment of other variables}

Required information about age, sex, marital status, place of residence, education, occupation, smoking status, use of supplements, family history of cancer and glioma, history of allergy and head trauma, history of hypertension, exposure to chemicals in the past 10 years, cooking methods, drug use, personal hair dye use, duration of cell phone use and history of exposure to the radiographic X-ray were examined using a pretested questionnaire. Participants' physical activity during the previous year was assessed using the International Physical Activity Questionnaire and expressed as metabolic equivalents (MET)-h/week. Subjects' weight was quantified by a dietitian using a digital scale to the nearest $500 \mathrm{~g}$ with the subjects wearing light clothing and no shoes. Height was measured in a standing position with a tape measure to the nearest $0.5 \mathrm{~cm}$ with the shoulders in normal position. BMI was calculated as weight (kg) divided by height $\left(\mathrm{m}^{2}\right)$.

On the basis of previous publications ${ }^{(25)}$, we considered farmers as having a high-risk occupation for glioma. Individuals who lived in places near the electromagnetic fields and cell phone and broadcast antennas in the last 10 years were defined as living in high-risk areas ${ }^{(26)}$. Individuals who consumed fried food at least twice per week were considered as frequent fried food users. This definition was also applied for barbecue use, microwave use as well as consumption of canned foods.

\section{Statistical methods}

General characteristics and dietary intakes of cases and controls were assessed using independent samples Student's $t$ test. To assess the association of the DASH-style diet with glioma, participants were categorised into tertiles based on their total score of the DASH diet. General characteristics of study participants across tertiles of the DASH diet score were compared using one-way ANOVA for continuous variables and $\chi^{2}$ test for categorical variables. To examine age-, sex- and energyadjusted intakes of foods and nutrients across tertiles of the DASH diet score, we applied ANCOVA. Association of the DASH diet and its components with glioma was assessed using logistic regression in different models. First, we controlled for age (continuous), sex (male/female) and energy intake $(\mathrm{kJ} / \mathrm{d}$ or $\mathrm{kcal} / \mathrm{d}$ ). Further adjustments were made for physical activity (continues), family history of cancer (yes/no), family history of glioma (yes/no), marital status (married/single/divorced), education (university graduated/ non-university education), highrisk occupation (farmer/non-farmer), high-risk residential area (yes/no), duration of cell phone use (continuous), supplement use (yes/no), history of exposure to the radiographic X-ray (yes/no), history of head trauma (yes/no), history of allergy (yes/no), history of hypertension (yes/no), smoking (smoker/ non-smoker), exposure to chemicals (yes/no), drug use (yes/ no), personal hair dye use (yes/no), frequent fried food intake (yes/no), frequent use of barbecue, canned foods and microwave (yes/no) in the second model. Additional controlling was performed for BMI in the last model. All confounders were chosen based on earlier publications. When examining the association of components of the DASH diet with glioma, an additional model controlling for the mutual effects of other components of the DASH-style diet was also constructed. In all these analyses, the bottom tertile of the DASH diet score or its components was considered as reference. The overall trend of OR across increasing tertiles of the DASH diet score or its components was examined by considering the median score in each category as a continuous variable. All the statistical analyses were carried out using SPSS (SPSS Inc., version 18). $P$ values were considered significant at $<0 \cdot 05$.

\section{Results}

General characteristics of the study participants across tertiles of the DASH diet score as well as separately by case and control groups are presented in Table 1 . Cases with glioma were more likely to have high-risk occupations, live in high-risk areas, be frequently exposed to chemicals and radiographic X-ray and have a family history of brain tumours compared with controls. Head trauma and smoking were more prevalent among them compared with controls. Individuals with glioma were less likely to use hair dye in the last 10 years compared with controls. Mean age and BMI were not significantly different between the two groups. Distribution of participants in terms of sex, education, family history of cancer, supplement use, history of allergy and hypertension was not significantly different between cases and controls. When examined across tertiles of the DASH diet score, we found no significant difference in mean age and BMI as well as in the distribution of participants in terms of categorical variables, except for the use of microwave $(P=0.01)$ and canned foods $(P=0 \cdot 007)$.

Dietary intakes of patients with glioma and those in the control group are provided in Table 2 . These intakes have also been examined across tertiles of the DASH diet score. Compared with controls, cases had higher intakes of $\mathrm{Na}$, refined grains, red and processed meats and partially hydrogenated 
Table 1. General characteristics of the study participants across case and control groups as well as across tertiles of the Dietary Approaches to Stop Hypertension (DASH) diet score

(Mean values and standard deviations)

\begin{tabular}{|c|c|c|c|c|c|c|c|c|c|c|c|c|}
\hline & \multicolumn{4}{|c|}{ Groups } & \multirow[b]{3}{*}{$P^{*}$} & \multicolumn{6}{|c|}{ Tertiles of the DASH diet score } & \multirow[b]{3}{*}{$P^{*}$} \\
\hline & \multicolumn{2}{|c|}{ Cases ( $n$ 128) } & \multicolumn{2}{|c|}{ Controls ( $n$ 256) } & & \multicolumn{2}{|c|}{$1(n 131)$} & \multicolumn{2}{|c|}{$2(n 139)$} & \multicolumn{2}{|c|}{$3(n 114)$} & \\
\hline & Mean & SD & Mean & SD & & Mean & SD & Mean & SD & Mean & SD & \\
\hline Age (years) & 43.4 & $14 \cdot 6$ & $42 \cdot 7$ & $13 \cdot 3$ & 0.65 & $42 \cdot 0$ & $13 \cdot 3$ & 43.7 & $14 \cdot 0$ & $43 \cdot 0$ & 13.9 & 0.61 \\
\hline Females (\%) & \multicolumn{2}{|c|}{41} & \multicolumn{2}{|c|}{42} & 0.99 & \multicolumn{2}{|c|}{37} & \multicolumn{2}{|c|}{45} & \multicolumn{2}{|c|}{44} & 0.33 \\
\hline BMI $\left(\mathrm{kg} / \mathrm{m}^{2}\right)$ & $26 \cdot 2$ & $4 \cdot 2$ & $26 \cdot 1$ & 3.8 & 0.75 & $26 \cdot 4$ & 4.49 & $26 \cdot 2$ & 3.6 & $25 \cdot 8$ & 3.7 & 0.49 \\
\hline Married (\%) & \multicolumn{2}{|c|}{79} & \multicolumn{2}{|c|}{80} & 0.66 & \multicolumn{2}{|c|}{80} & \multicolumn{2}{|c|}{80} & \multicolumn{2}{|c|}{78} & 0.91 \\
\hline University graduated (\%) & \multirow{2}{*}{\multicolumn{2}{|c|}{12}} & \multirow{2}{*}{\multicolumn{2}{|c|}{$\begin{array}{c}17 \\
3\end{array}$}} & 0.22 & \multirow{2}{*}{\multicolumn{2}{|c|}{$\begin{array}{r}11 \\
8\end{array}$}} & \multicolumn{2}{|c|}{17} & \multicolumn{2}{|c|}{18} & 0.21 \\
\hline High-risk jobs† (\%) & & & & & 0.003 & & & & 4 & & 3 & 0.11 \\
\hline High-risk residential areał (\%) & \multicolumn{2}{|c|}{30} & \multicolumn{2}{|c|}{21} & 0.05 & & & & 5 & & 28 & 0.29 \\
\hline Duration of cell phone use (years) & 2.85 & $2 \cdot 8$ & 3.70 & 2.56 & 0.003 & 3.21 & 2.49 & 3.38 & 2.69 & 3.74 & 2.93 & 0.30 \\
\hline History of exposure to the radiographic X-ray (\%) & $1 \epsilon$ & & & & 0.01 & & & & 9 & & 1 & 0.73 \\
\hline History of head trauma $(\%)$ & 4 & & & & 0.004 & & & & 6 & & 38 & 0.22 \\
\hline History of allergy (\%) & 25 & & & & 0.40 & & & & 2 & & 28 & 0.22 \\
\hline History of hypertension (\%) & 2 & & & 5 & 0.28 & & 5 & & 4 & & 3 & 0.91 \\
\hline Smoking status (\%) & & & & & 0.003 & & & & & & & 0.13 \\
\hline Non-smoker & 8 & & & & & & & & 8 & & 34 & \\
\hline$<5 \mathrm{n} / \mathrm{d}$ & 2 & & & 8 & & & 6 & & 5 & & 7 & \\
\hline $5-10 \mathrm{n} / \mathrm{d}$ & 2 & & & 7 & & & 9 & & 4 & & 1 & \\
\hline $11-15 \mathrm{n} / \mathrm{d}$ & 5 & & & 6 & & & 5 & & 7 & & 6 & \\
\hline $16-20 \mathrm{n} / \mathrm{d}$ & 5 & & & 4 & & & 6 & & 5 & & 1 & \\
\hline$>20 \mathrm{n} / \mathrm{d}$ & 2 & & & o & & & 1 & & 1 & & 1 & \\
\hline Frequent fried food intake§ (\%) & 9 & & & & 0.001 & & & & 3 & & 33 & 0.86 \\
\hline Frequent use of barbecuell (\%) & 16 & & & & 0.21 & & & & 4 & & 4 & 0.89 \\
\hline Frequent microwave usell (\%) & $\varepsilon$ & & & & 0.002 & & 9 & & 5 & & 22 & 0.01 \\
\hline Frequent canned foods intakell (\%) & $\epsilon$ & & & 7 & 0.52 & & 3 & & 4 & & 2 & 0.007 \\
\hline Drug use $(\%)$ & $\varepsilon$ & & & 5 & 0.36 & & 5 & & 4 & & 0 & $0 \cdot 11$ \\
\hline Personal hair dye use (\%) & 22 & & & & $<0.001$ & & & & 4 & & 12 & 0.12 \\
\hline Exposure to chemicals (\%) & 20 & & & & 0.01 & & & & 4 & & 15 & 0.67 \\
\hline Family history of glioma (\%) & 15 & & & 5 & $<0.001$ & & & & 1 & & 5 & 0.11 \\
\hline Family history of cancer (\%) & 33 & & & & 0.90 & & & & 7 & & 37 & 0.13 \\
\hline Supplement use (\%) & $\varepsilon$ & & & & 0.36 & & 0 & & 1 & & 9 & 0.54 \\
\hline Physical activity (MET-h/week) & 34.7 & $6 \cdot 3$ & 33.8 & 5.5 & 0.12 & 34.7 & $5 \cdot 8$ & 33.9 & $6 \cdot 0$ & 33.7 & 5.4 & 0.39 \\
\hline
\end{tabular}

MET, metabolic equivalents.

* $P$ values were obtained from independent Student's $t$ test, one-way ANOVA or $\chi^{2}$ test, where appropriate.

† Farmers were considered as having a high-risk occupation.

¥ Individuals who lived in places near electromagnetic fields and cell phone and broadcast antennas in the last 10 years were defined as living in high-risk areas.

$\S$ Individuals who consumed fried food at least twice per week were considered as frequent fried food users.

II Individuals who used barbecue, microwave and canned foods at least twice per week were considered as frequent users.

vegetable oils and lower intakes of fats, $\mathrm{Ca}$, Se, vitamin $\mathrm{E}$, whole-grains, fruits, vegetables, dairy products, legumes and nuts, and non-hydrogenated vegetable oils. Adherence to the DASH diet was associated with greater intakes of $\mathrm{K}, \mathrm{Ca}$, Se, folate, vitamin $\mathrm{C}$, dietary fibre, fish, fruits, vegetables, dairy products, and legumes and nuts. Individuals in the top tertile of the DASH diet had lower intakes of fat, SFA, Na, refined grains, red and processed meats, salt and sugar-sweetened beverages compared with those in the bottom tertile.

Multivariable-adjusted OR for glioma across tertiles of the DASH diet score are shown in Table 3. After controlling for age, sex and energy intake, individuals with the greatest adherence to the DASH diet were less likely to have glioma compared with those with the lowest adherence - such that those in the top tertile were $62 \%$ less likely to have glioma than those in the bottom tertile (95\% CI 0.21, 0.66). Further adjustment for other potential confounders strengthened the association (OR for the highest tertile $v$. the lowest: OR 0.26 ; $95 \%$ CI $0 \cdot 13,0.54$ ). Additional controlling for BMI did not influence the association significantly (OR 0.28; $95 \%$ CI 0.13, 0.57).
Multivariable-adjusted OR for glioma across tertiles of the components of the DASH diet score are provided in Table 4. After controlling for potential confounders, individuals with the highest consumption of fruits had lower odds for having glioma compared with those with the lowest intake (OR 0.30; 95\% CI 0.15, 0.59). Additional controlling for dietary intakes of other food groups did not affect this association (OR 0.31; $95 \%$ CI 0.14, 0.68). Even after further adjustment of BMI, this association remained significant (OR 0.31; $95 \%$ CI 0.14, 0.69). A protective association was also observed between consumption of legumes and nuts and risk of glioma - such that after controlling for potential confounders and intake of other food groups, those in the top tertile of legumes and nuts intake were $77 \%$ less likely to have glioma compared with those in the bottom tertile (OR 0.23; $95 \%$ CI 0.10, $0.53)$. Further controlling for BMI did not affect this association (OR 0.24; 95\% CI 0.11, 0.55). We found a significant positive association between red and processed meat intakes and risk of glioma. After adjustment for potential confounders including dietary intakes of other food groups and BMI, those with the greatest intake of red and processed meat had 2.60 times greater 
Table 2. Dietary intakes of study participants across case and control groups as well as across tertiles of the Dietary Approaches to Stop Hypertension (DASH) diet score*

(Mean values and standard deviations)

\begin{tabular}{|c|c|c|c|c|c|c|c|c|c|c|c|c|}
\hline & \multicolumn{4}{|c|}{ Groups } & \multirow[b]{3}{*}{$P$} & \multicolumn{6}{|c|}{ Tertiles of the DASH diet score } & \multirow[b]{3}{*}{$P$} \\
\hline & \multicolumn{2}{|c|}{ Cases ( $n$ 128) } & \multicolumn{2}{|c|}{ Controls ( $n$ 256) } & & \multicolumn{2}{|c|}{$1(n 131)$} & \multicolumn{2}{|c|}{$2(n 139)$} & \multicolumn{2}{|c|}{$3(n 114)$} & \\
\hline & Mean & SD & Mean & SD & & Mean & SD & Mean & SD & Mean & SD & \\
\hline Energy (kJ/d) & 10795 & 2343 & 10715 & 3021 & & 10983 & 2787 & 10682 & 3904 & 11309 & 2544 & \\
\hline Energy (kcal/d) & 2580 & 560 & 2561 & 722 & 0.79 & 2625 & 666 & 2553 & 933 & 2703 & 608 & 0.72 \\
\hline \multicolumn{13}{|l|}{ Nutrients } \\
\hline Proteins (g/d) & 98 & 22 & 97 & 30 & 0.70 & 96 & 22 & 98 & 47 & 103 & 36 & 0.37 \\
\hline Fats $(\mathrm{g} / \mathrm{d})$ & 62 & 19 & 66 & 22 & 0.05 & 68 & 19 & 65 & 22 & 60 & 19 & 0.02 \\
\hline SFA $(g / d)$ & 19 & 7 & 21 & 9 & 0.08 & 22 & 9 & 21 & 9 & 17 & 7 & 0.04 \\
\hline $\mathrm{Na}(\mathrm{mg} / \mathrm{d})$ & 4823 & 1506 & 4320 & 1586 & 0.004 & 5892 & 1287 & 4544 & 1406 & 4204 & 1976 & 0.005 \\
\hline $\mathrm{K}(\mathrm{mg} / \mathrm{d})$ & 4074 & 783 & 6363 & 1423 & 0.03 & 3993 & 1267 & 4198 & 1183 & 4674 & 1226 & $<0.001$ \\
\hline $\mathrm{Ca}(\mathrm{mg} / \mathrm{d})$ & 1019 & 263 & 1139 & 358 & 0.001 & 842 & 273 & 1105 & 371 & 1195 & 326 & $<0.001$ \\
\hline $\mathrm{Se}(\mathrm{mcg} / \mathrm{d})$ & 0.06 & 0.04 & 0.08 & 0.36 & 0.02 & 0.03 & 0.04 & 0.06 & 0.34 & 0.08 & 0.03 & 0.04 \\
\hline Vitamin E (mg/d) & 5 & 2 & 6 & 3 & 0.03 & 4 & 3 & 5 & 3 & 8 & 2 & 0.18 \\
\hline Vitamin $B_{6}(\mathrm{mg} / \mathrm{d})$ & 2 & 0.54 & 2 & 0.76 & 0.13 & 2 & 0.7 & 2 & 0.59 & 2 & 0.79 & 0.06 \\
\hline Folate $(\mathrm{mcg} / \mathrm{d})$ & 349 & 90 & 382 & 302 & 0.23 & 248 & 116 & 283 & 390 & 389 & 113 & 0.04 \\
\hline Vitamin C (mg/d) & 126 & 59 & 143 & 113 & $0 \cdot 11$ & 115 & 145 & 135 & 58 & 154 & 51 & 0.04 \\
\hline Dietary Fibre $(\mathrm{g} / \mathrm{d})$ & 23 & 11 & 23 & 15 & 0.82 & 13 & 14 & 19 & 15 & 27 & 8 & 0.03 \\
\hline \multicolumn{13}{|l|}{ Food groups } \\
\hline Refined grains (g/d) & 501 & 175 & 421 & 182 & $<0.001$ & 509 & 185 & 431 & 183 & 398 & 163 & $<0.001$ \\
\hline Whole-grains (g/d) & 150 & 134 & 177 & 108 & 0.03 & 152 & 136 & 154 & 102 & 174 & 114 & 0.28 \\
\hline White meats $(\mathrm{g} / \mathrm{d})$ & 30 & 13 & 33 & 22 & 0.23 & 30 & 13 & 30 & 13 & 35 & 30 & 0.07 \\
\hline Red meats $(\mathrm{g} / \mathrm{d})$ & 41 & 28 & 36 & 20 & 0.03 & 46 & 20 & 36 & 22 & 32 & 25 & $<0.001$ \\
\hline Fish $(g / d)$ & 9 & 12 & 9 & 9 & 0.86 & 8 & 11 & 9 & 9 & 11 & 11 & 0.05 \\
\hline Fruits $(\mathrm{g} / \mathrm{d})$ & 325 & 99 & 361 & 124 & 0.005 & 299 & 93 & 354 & 119 & 400 & 119 & $<0.001$ \\
\hline Vegetables $(\mathrm{g} / \mathrm{d})$ & 258 & 83 & 274 & 86 & 0.07 & 229 & 62 & 262 & 75 & 323 & 93 & $<0.001$ \\
\hline Dairy products $(\mathrm{g} / \mathrm{d})$ & 309 & 117 & 355 & 131 & 0.001 & 289 & 120 & 346 & 126 & 389 & 121 & $<0.001$ \\
\hline Legumes and nuts $(\mathrm{g} / \mathrm{d})$ & 40 & 23 & 46 & 20 & 0.01 & 36 & 18 & 43 & 20 & 55 & 21 & $<0.001$ \\
\hline Salt $(g / d)$ & 6 & 2 & 6 & 2 & 0.01 & 7 & 2 & 6 & 2 & 4 & 2 & 0.007 \\
\hline $\begin{array}{l}\text { Sugar-sweetened beverages } \\
(\mathrm{g} / \mathrm{d})\end{array}$ & 79 & 67 & 83 & 74 & 0.57 & 116 & 54 & 79 & 66 & 55 & 83 & $<0.001$ \\
\hline $\begin{array}{l}\text { Partially hydrogenated } \\
\text { vegetable oils }(\mathrm{g} / \mathrm{d})\end{array}$ & 15 & 15 & 9 & 12 & $<0.001$ & 12 & 15 & 10 & 13 & 8 & 13 & 0.19 \\
\hline $\begin{array}{l}\text { Non-hydrogenated vegetable } \\
\text { oils }(\mathrm{g} / \mathrm{d})\end{array}$ & 7 & 6 & 8 & 5 & 0.03 & 7 & 5 & 8 & 5 & 8 & 5 & 0.34 \\
\hline
\end{tabular}

* All values were adjusted for age sex and energy, except for dietary energy intake, which was only adjusted for age and sex. Comparisons were made using ANOVA.

Table 3. Multivariable-adjusted ratios for glioma across different categories of the Dietary Approaches to Stop Hypertension (DASH) diet score* (Odds ratios and $95 \%$ confidence intervals)

\begin{tabular}{|c|c|c|c|c|c|c|}
\hline & \multicolumn{5}{|c|}{ Tertiles of the DASH diet score } & \multirow[b]{3}{*}{$P_{\text {trend }}$} \\
\hline & \multirow{2}{*}{$\frac{1(n 131)}{\mathrm{OR}}$} & \multicolumn{2}{|c|}{$2(n 139)$} & \multicolumn{2}{|c|}{$3(n 114)$} & \\
\hline & & OR & $95 \% \mathrm{Cl}$ & OR & $95 \% \mathrm{Cl}$ & \\
\hline DASH Score range & $<21$ & \multicolumn{2}{|c|}{$21-25$} & \multicolumn{2}{|c|}{$>25$} & \\
\hline Number of cases & 59 & \multicolumn{2}{|c|}{41} & \multicolumn{2}{|c|}{27} & \\
\hline Crude & 1.00 & 0.51 & $0.30-0.84$ & 0.38 & $0.22-0.66$ & $<0.001$ \\
\hline Model 1† & 1.00 & 0.50 & $0.30-0.84$ & 0.38 & $0.21-0.66$ & $<0.001$ \\
\hline Model $2 \ddagger$ & 1.00 & 0.38 & $0.20-0.71$ & 0.26 & $0.13-0.54$ & $<0.001$ \\
\hline Model $3 \S$ & 1.00 & 0.39 & $0.21-0.73$ & 0.28 & $0.13-0.57$ & $<0.001$ \\
\hline \multicolumn{7}{|c|}{$\begin{array}{l}\text { * Binary logistic regression was used to obtain OR and } 95 \% \mathrm{Cl} \text {. The overall trend of OR across increasing tertiles was examined by considering the median score in each category } \\
\text { as a continuous variable. } \\
\text { † Model 1: adjusted for age (continuous), sex (male/female) and energy intake (kJ/d or kcal/d). } \\
\text { † Model 2: further adjustments were made for physical activity (continues), family history of cancer (yes/no), family history of glioma (yes/no), marital status (married/single/ } \\
\text { divorced), education (university graduated/ non-university education), high-risk occupation (farmer/ non-farmer), high-risk residential area (yes/no), duration of cell phone use } \\
\text { (continuous), supplement use (yes/no), history of exposure to the radiographic X-ray (yes/no), history of head trauma (yes/no), history of allergy (yes/no), history of hypertension } \\
\text { (yes/no), smoking status (smoker/non-smoker), exposure to chemicals (yes/no), drug use (yes/no), personal hair dye use, frequent fried food intake (yes/no), frequent use of } \\
\text { barbecue, canned foods and microwave (yes/no). } \\
\text { § Model 3: additionally adjusted for BMl (continuous). }\end{array}$} \\
\hline
\end{tabular}


Table 4. The association between components of the Dietary Approaches to Stop Hypertension (DASH) diet and glioma* (Odds ratios and $95 \%$ confidence intervals)

\begin{tabular}{|c|c|c|c|c|c|c|}
\hline & \multicolumn{5}{|c|}{ Tertiles of components of the DASH diet } & \multirow[b]{3}{*}{$P_{\text {trend }}$} \\
\hline & \multirow{2}{*}{$\frac{1(n \text { 131) }}{\mathrm{OR}}$} & \multicolumn{2}{|r|}{$2(n 139)$} & \multicolumn{2}{|c|}{$3(n 114)$} & \\
\hline & & OR & $95 \% \mathrm{Cl}$ & OR & $95 \% \mathrm{Cl}$ & \\
\hline \multicolumn{7}{|l|}{ Whole-grains } \\
\hline Range (g/d) & $<92$ & & $92-196$ & & & \\
\hline Number of cases & 39 & & 45 & & & \\
\hline Multivariable-adjusted $\dagger$ & 1.00 & 0.90 & $0.45-1.76$ & 1.26 & $0.66-2.40$ & 0.41 \\
\hline Multivariable-adjusted + other food groups & 1.00 & 1.33 & $0.63-2.79$ & 1.04 & $0.48-2 \cdot 26$ & 0.39 \\
\hline Multivariable-adjusted + other food groups + BMI & 1.00 & 1.34 & $0.64-2.82$ & 1.06 & $0.48-2 \cdot 30$ & 0.35 \\
\hline \multicolumn{7}{|l|}{ Fruits } \\
\hline Range (g/d) & $<305$ & & $305-385$ & & & \\
\hline Number of cases & 52 & & 43 & & & \\
\hline Multivariable-adjusted & 1.00 & 0.40 & $0.20-0.78$ & 0.30 & $0.15-0.59$ & 0.01 \\
\hline Multivariable-adjusted + other food groups & 1.00 & 0.38 & $0.17-0.84$ & 0.31 & $0.14-0.68$ & 0.001 \\
\hline Multivariable-adjusted + other food groups $+\mathrm{BMI}$ & 1.00 & 0.38 & $0.17-0.83$ & 0.31 & $0.14-0.69$ & 0.001 \\
\hline \multicolumn{7}{|l|}{ Vegetables } \\
\hline Range (g/d) & $<230$ & & $230-297$ & & & \\
\hline Number of cases & 47 & & 41 & & & \\
\hline Multivariable-adjusted & 1.00 & 0.75 & $0.40-1.41$ & 0.52 & $0.27-1.01$ & 0.07 \\
\hline Multivariable-adjusted + other food groups & 1.00 & 0.98 & $0.47-2.06$ & 0.65 & $0.28-1.51$ & $0 \cdot 11$ \\
\hline Multivariable-adjusted + other food groups + BMI & 1.00 & 1.00 & $0.48-2 \cdot 10$ & 0.66 & $0.28-1.53$ & 0.23 \\
\hline \multicolumn{7}{|l|}{ Dairy products } \\
\hline Range $(\mathrm{g} / \mathrm{d})$ & $<284$ & & $284-387$ & & & \\
\hline Number of cases & 56 & & 44 & & & \\
\hline Multivariable-adjusted & 1.00 & 0.81 & $0.43-1.52$ & 0.48 & $0.24-0.94$ & 0.001 \\
\hline Multivariable-adjusted + other food groups & 1.00 & 1.37 & $0.68-2.79$ & 0.55 & $0.25-1.21$ & 0.19 \\
\hline Multivariable-adjusted + other food groups $+\mathrm{BMI}$ & 1.00 & 1.41 & $0.69-2.88$ & 0.56 & $0.26-1.24$ & 0.35 \\
\hline \multicolumn{7}{|l|}{ Legumes and Nuts } \\
\hline Range $(\mathrm{g} / \mathrm{d})$ & $<33$ & & $33-51$ & & & \\
\hline Number of cases & 57 & & 36 & & & \\
\hline Multivariable-adjusted & 1.00 & 0.26 & $0.12-0.52$ & 0.20 & $0.10-0.41$ & $<0.001$ \\
\hline Multivariable-adjusted + other food groups & 1.00 & 0.21 & $0.09-0.46$ & 0.23 & $0.10-0.53$ & $<0.001$ \\
\hline Multivariable-adjusted + other food groups + BMI & 1.00 & 0.21 & $0.09-0.46$ & 0.24 & $0.11-0.55$ & $<0.001$ \\
\hline \multicolumn{7}{|l|}{ Meats and processed meats } \\
\hline Range $(g / d)$ & $<29$ & & $29-42$ & & & \\
\hline Number of cases & 45 & & 33 & & & \\
\hline Multivariable-adjusted & 1.00 & 0.82 & $0.42-1.61$ & 1.91 & $0.96-3.80$ & $0 \cdot 19$ \\
\hline Multivariable-adjusted + other food groups & 1.00 & 1.07 & $0.50-2.29$ & 2.53 & $1 \cdot 13-5 \cdot 65$ & 0.05 \\
\hline Multivariable-adjusted + other food groups + BMI & 1.00 & 1.07 & $0.50-2.29$ & $2 \cdot 60$ & $1 \cdot 16-5 \cdot 81$ & 0.04 \\
\hline \multicolumn{7}{|l|}{ Salt } \\
\hline Range (g/d) & $<5.1$ & & $5 \cdot 1-7 \cdot 0$ & & & \\
\hline Number of cases & 31 & & 46 & & & \\
\hline Multivariable-adjusted & 1.00 & 1.98 & $1.01-3.85$ & 2.43 & $1.22-4.83$ & 0.01 \\
\hline Multivariable-adjusted + other food groups & 1.00 & $2 \cdot 26$ & $1.07-4.78$ & 2.93 & $1.33-6.44$ & 0.001 \\
\hline Multivariable-adjusted + other food groups + BMI & 1.00 & $2 \cdot 17$ & $1.02-4.62$ & $2 \cdot 87$ & $1.30-6.34$ & 0.001 \\
\hline \multicolumn{7}{|l|}{ Sweats and sweetened beverages } \\
\hline Range $(\mathrm{g} / \mathrm{d})$ & $<49$ & & 49-91 & & & \\
\hline Number of cases & 49 & & 34 & & & \\
\hline Multivariable-adjusted & 1.00 & 0.59 & $0.30-1.15$ & 0.83 & $0.43-1.61$ & 0.21 \\
\hline Multivariable-adjusted + other food groups & 1.00 & 0.56 & $0.26-1 \cdot 18$ & 0.81 & $0.35-1.88$ & 0.24 \\
\hline Multivariable-adjusted + other food groups + BMI & 1.00 & 0.56 & $0.26-1.17$ & 0.80 & $0.34-1.86$ & 0.34 \\
\hline
\end{tabular}

* Binary logistic regression was used to obtain OR and $95 \% \mathrm{Cl}$. The overall trend of the OR across increasing tertiles was examined by considering the median score in each category as a continuous variable.

† Adjusted for age (continuous), sex (male/female) and energy intake (kJ/d or kcal/d), physical activity (continues), family history of cancer (yes/no), family history of glioma (yes/no), marital status (married/single/divorced), education (university graduated/non-university education), high-risk occupation (farmer/ non-farmer), high-risk residential area (yes/no), duration of cell phone use (continuous), supplement use (yes/no), history of exposure to the radiographic X-ray (yes/no), history of head trauma (yes/no), history of allergy (yes/ no), history of hypertension (yes/no), smoking status (smoker/non-smoker), exposure to chemicals (yes/no), drug use (yes/no), personal hair dye use, frequent fried food intake (yes/no), frequent use of barbecue, canned foods and microwave (yes/no).

chance to have glioma than those with the lowest intake (95\% CI $1 \cdot 16,5 \cdot 81)$. This was also the case for salt intake. Individuals in the highest tertile of salt intake were 2.87 times more likely to have glioma than those in the bottom tertile after taking all potential confounders into account (OR 2·87; $95 \%$ CI 1·30, 6·34).

\section{Discussion}

We found an inverse relationship between adherence to the DASH-style dietary pattern and odds of having glioma. In addition, some components of the DASH-style diet including 
fruits, legumes and nuts as well as salt and red meat intake were also significantly associated with the risk. These associations persisted in multivariate models accounting for several confounders. To the best of our knowledge, this study is the first to examine the association between adherence to the DASH-style diet and gliomas.

DASH eating plan was initially suggested for the management of hypertension $^{(12,13)}$; however, its beneficial effects were then demonstrated for obesity ${ }^{(14)}$, the metabolic syndrome ${ }^{(15)}$, gestational diabetes $^{(16)}$ and some cancers ${ }^{(17)}$. In the present case-control study, we found that adherence to the DASH-style diet was inversely associated with the odds of glioma. Although several case-control studies or meta-analyses have investigated the association between several dietary components and risk of glioma $^{(5-8)}$, limited information is available linking whole dietary patterns to the risk $^{(9)}$. In a case-control study in Brazil ${ }^{(9)}$, the snack pattern and animal fat dietary pattern, rich in sugar and fats, during adolescence were associated with the lowest odds of developing brain tumours in adults. No significant association was observed between the traditional and mixed dietary patterns in adolescence and risk of brain tumours in adulthood $^{(9)}$. Unlike the present study that used a priori method, the investigators in Brazil ${ }^{(9)}$ used a posteriori approach to identify dietary patterns. In addition, several confounders that have been taken into account in the present analysis have not been adjusted for in the Brazilian study ${ }^{(9)}$. These differences might explain the different findings of these two studies. Dietary intakes consist of combinations of nutrients and foods. Therefore, the dietary pattern approach, which allows holistic assessment of diet and the relations with the outcome, would provide much more information beyond those identified by foods and nutrients ${ }^{(10)}$.

We found an inverse association between consumption of fruits, legumes and nuts and risk of glioma. In addition, dietary intakes of red meat and salt were associated with greater risk of glioma in the present study. Despite the lack of any evidence about the association of the DASH-style diet with glioma, several studies have reported the association of individual components of the DASH diet with glioma. In line with our findings, several other case-control studies have found an inverse association between fruit consumption and the risk of glioma ${ }^{(27,28)}$. However, some other case-control studies have failed to find any significant association ${ }^{(29,30)}$ or have even reached conclusions about a positive association between fruit intake and risk of glioma ${ }^{(31,32)}$. Different findings might be explained by different study designs, lack of controlling for potential confounders or difference in dietary assessment tools and considering different types of brain tumours.

The protective association of fruit intake against glioma might be explained by the high vitamin $\mathrm{C}$ content of this food group. Vitamin $\mathrm{C}$ has been well known as a nitrosation inhibitor ${ }^{(32)}$. Several studies have shown an inverse association between dietary antioxidants intake and risk of glioma ${ }^{(3,4)}$. The inverse association between legumes and nuts intake and risk of glioma has also been shown by some previous case-control publications $^{(33)}$. Such protective association might be explained by the high content of inositol hexaphosphate in nuts, as shown in previous studies ${ }^{(34)}$. Although some case-control studies, consistent with ours, have demonstrated a positive association between red meat intake and glioma ${ }^{(6)}$, others have found this association only for cured meats ${ }^{(35,36)}$ or only for non-cured meats ${ }^{(32)}$. The red meat category in the present study was composed of both processed and unprocessed meats due to low consumption of processed meats in the study population. Therefore, it is difficult to separate their effects from each other. However, when we considered only unprocessed red meat intake, the associations did not alter. Therefore, it seems that some factors beyond $N$-nitroso compounds of meat might contribute to the risk. Additional studies are required to examine the possible synergistic effect of meats and nitrosationinhibiting foods. Taken together, it seems that assessment of the association of individual foods and food groups to the risk of glioma might result in the missing of the effect of other foods and nutrients, and therefore further studies assessing the association of whole dietary patterns and risk of glioma are needed to clarify the diet-glioma relations.

This study has several strengths. This was the first study to examine the association between adherence to the DASH diet and glioma. We controlled for a wide range of confounders in the present study to reach an independent association between the DASH-style eating pattern and risk of glioma. In addition, patients enrolled in the study were new cases of glioma who had been diagnosed with this malignancy in the past 1 month. This reduces the possibility of changing usual dietary intakes in these patients. It must also be kept in mind that the study comes from the under-studied region of the Middle East where available information on diet-disease relations is very limited.

However, some limitations also need to be taken into account when interpreting our findings. The first is the case-control design of the study that is subject to several biases including selection and recall bias. Owing to such design of the study, one cannot confer causality. Although case-control studies are efficient in terms of time and cost, they are highly susceptible to both selection and recall biases. Recall bias, in which cases may recall their past diet differently in the context of their cancer diagnosis, is problematic because dietary assessment occurs after diagnosis. An additional concern for case-control studies of diet is that cases may have altered their diet before diagnosis due to early symptoms of the disease. In addition, cases in the present study were selected from hospitals; therefore, identified risk factors may be unique to these hospitals. This methodological choice may weaken the generalisability of the study findings. However, case-control studies are suitable for studying the association of diet with rare disease with long latency period including cancers. To account for recall bias in this study design, we enrolled hospital-based controls to report exposures in a year before enrollment. Second, as with all epidemiological studies that apply FFQ, misclassification of study participants is unavoidable. However, we used energy-adjusted intakes of all food groups to construct the DASH diet score, which could in turn reduce the possibility of subject misclassification. Third, all food groups in the DASH-style dietary pattern were given an equal weight; however, some foods might influence the risk differently. Fourth, although we controlled for several confounders, one cannot exclude the possibility of residual confounding. Fifth, the study findings cannot easily be 
extrapolated to the general population due to several methodological concerns in case-control designs. However, participants in the study were selected from referral hospitals in the country, covering people with diverse socio-economic status. Therefore, their dietary intakes could cover a wide range of dietary habits. In addition, as the dietary intakes of Middle Eastern population are different from Western nations, the generalisability of the findings to other populations should be made cautiously. As salt intake was not measured satisfactorily with an FFQ, scoring by quintiles would be least prone to misclassification.

On the basis of this case-control study, we found that adherence to the DASH-style dietary pattern was inversely associated with glioma. In addition, some components of the DASH diet, including red meats and salt intake, were positively associated with glioma. Consumption of nuts and legumes as well as fruits was inversely associated with glioma. Prospective cohort studies are required to confirm our findings.

\section{Acknowledgements}

This study was extracted from an M.S. dissertation, which was approved by the School of Nutrition \& Food Sciences, Isfahan University of Medical Sciences (no. 394288). The financial support for this study was from Food Security Research Center, Isfahan University of Medical Sciences, Isfahan, Iran.

This study was financially supported by the Research Council of the Food Security Research Center, Isfahan University of Medical Sciences, Isfahan, Iran.

S. B.-K., M. S., M. M., H. T., G. S., P. S. and A. E. contributed to the conception, design, data collection, statistical analyses, data interpretation, manuscript drafting, approval of the final version of the manuscript and agreed for all aspects of the work.

None of the authors declared potential personal or financial conflicts of interest.

\section{References}

1. Ohgaki H \& Kleihues P (2005) Population-based studies on incidence, survival rates, and genetic alterations in astrocytic and oligodendroglial gliomas. J Neuropathol Exp Neurol 64 , 479-489.

2. Alimohamadi SM, Ghodsi SM \& Ketabchi SE (2008) Epidemiologic patterns of primary brain tumors in Iran. Asian Pac J Cancer Prev 9, 361-362.

3. Sheweita SA \& Sheikh BY (2011) Can dietary antioxidants reduce the incidence of brain tumors? Curr Drug Metab 12, 587-593.

4. Kyritsis AP, Bondy ML \& Levin VA (2011) Modulation of glioma risk and progression by dietary nutrients and antiinflammatory agents. Nutr Cancer 63, 174-184.

5. Wei Y, Zou D, Cao D, et al. (2015) Association between processed meat and red meat consumption and risk for glioma: a meta-analysis from 14 articles. Nutrition 31, $45-50$.

6. Boeing H, Schlehofer B, Blettner M, et al. (1993) Dietary carcinogens and the risk for glioma and meningioma in Germany. Int J Cancer 53, 561-565.

7. Li Y (2014) Association between fruit and vegetable intake and risk for glioma: a meta-analysis. Nutrition 30, 1272-1278.
8. Hu J, La Vecchia C, Negri E, et al. (1999) Diet and brain cancer in adults: a case-control study in northeast China. Int J Cancer 81, 20-23.

9. Albuquerque RCR, Monteiro GTR, Pereira RA, et al. (2012) Dietary patterns and brain cancer: a case-control study in Rio de Janeiro, Brazil. Cad Saude Colet 20, 271-279.

10. Hu FB (2002) Dietary pattern analysis: a new direction in nutritional epidemiology. Curr Opin Lipidol 13, 3-9.

11. Vogt TM, Appel LJ, Obarzanek E, et al. (1999) Dietary Approaches to Stop Hypertension: rationale, design, and methods. DASH Collaborative Research Group. J Am Diet Assoc 99, S12-S18.

12. Appel LJ, Brands MW, Daniels SR, et al. (2006) Dietary approaches to prevent and treat hypertension: a scientific statement from the American Heart Association. Hypertension 47, 296-308.

13. Saneei P, Salehi-Abargouei A, Esmaillzadeh A, et al. (2014) Influence of Dietary Approaches to Stop Hypertension (DASH) diet on blood pressure: a systematic review and metaanalysis on randomized controlled trials. Nutr Metab Cardiovasc Dis 24, 1253-1261.

14. Barak F, Falahi E, Keshteli AH, et al. (2015) Adherence to the Dietary Approaches to Stop Hypertension (DASH) diet in relation to obesity among Iranian female nurses. Public Health Nutr 18, 705-712.

15. Saneei P, Fallahi E, Barak F, et al. (2015) Adherence to the DASH diet and prevalence of the metabolic syndrome among Iranian women. Eur J Nutr 54, 421-428.

16. Asemi Z, Tabassi Z, Samimi M, et al. (2013) Favourable effects of the Dietary Approaches to Stop Hypertension diet on glucose tolerance and lipid profiles in gestational diabetes: a randomised clinical trial. Br J Nutr 109, 2024-2030.

17. Jones-McLean E, Hu J, Greene-Finestone LS, et al. (2015) A DASH dietary pattern and the risk of colorectal cancer in Canadian adults. Health Promot Chronic Dis Prev Can 35, $12-20$.

18. Esmaillzadeh A \& Azadbakht L (2008) Major dietary patterns in relation to general obesity and central adiposity among Iranian women. J Nutr 138, 358-363.

19. Bahreynian M \& Esmaillzadeh A (2012) Quantity and quality of carbohydrate intake in Iran: a target for nutritional intervention. Arch Iran Med 15, 648-649.

20. Malekshah AF, Kimiagar M, Saadatian-Elahi M, et al. (2006) Validity and reliability of a new food frequency questionnaire compared to $24 \mathrm{~h}$ recalls and biochemical measurements: pilot phase of Golestan cohort study of esophageal cancer. Eur J Clin Nutr 60, 971-977.

21. Ghaffarpour M, Houshiar-Rad A \& Kianfar H (1999) The Manual for Household Measures, Cooking Yields Factors and Edible Portion of Foods. Tehran: Keshaverzi Press; in Farsi.

22. US Department of Agriculture. National nutrient database for standard reference release 17 (database on the Internet). USDA, Washington, DC. http://www.nal.usda.gov/fnic/ foodcomp (accessed March 2015).

23. Fung TT, Hu FB, Wu K, et al. (2010) The Mediterranean and Dietary Approaches to Stop Hypertension (DASH) diets and colorectal cancer. Am J Clin Nutr 92, 1429-1435.

24. Willett WC, Howe GR \& Kushi LH (1997) Adjustment for total energy intake in epidemiologic studies. Am J Clin Nutr $\mathbf{6 5}$, 1220S-1228S.

25. Ruder AM, Carreón T, Butler MA, et al. (2009) Exposure to farm crops, livestock, and farm tasks and risk of glioma: the Upper Midwest Health Study. Am J Epidemiol 169, 1479-1491.

26. Morgan LL, Miller AB, Sasco A, et al. (2015) Mobile phone radiation causes brain tumors and should be classified as a 
probable human carcinogen (2A) (review). Int J Oncol $\mathbf{4 6}$ 1865-1871.

27. Preston-Martin S, Yu MC, Henderson BE, et al. (1983) Risk factors for meningiomas in men in Los Angeles County. J Natl Cancer Inst 70, 863-866.

28. Preston-Martin S \& Mack W (1991) Gliomas and meningiomas in men in Los Angeles County: investigation of exposures to N-nitroso compounds. IARC Sci Publ 105, 197-203.

29. Chen H, Ward MH, Tucker KL, et al. (2002) Diet and risk of adult glioma in eastern Nebraska, United States. Cancer Causes Control 13, 647-655.

30. Burch JD, Craib KJ, Choi BC, et al. (1987) An exploratory casecontrol study of brain tumors in adults. J Natl Cancer Inst $\mathbf{7 8}$, 601-609.

31. Kaplan S, Novikov I \& Modan B (1997) Nutritional factors in the etiology of brain tumors: potential role of nitrosamines, fat, and cholesterol. Am J Epidemiol 146, 832-841.
32. Terry MB, Howe G, Pogoda JM, et al. (2009) An international case-control study of adult diet and brain tumor risk: a histology-specific analysis by food group. Ann Epidemiol 19, 161-171.

33. Giles GG, McNeil JJ, Donnan G, et al. (1994) Dietary factors and the risk of glioma in adults: results of a case-control study in Melbourne, Australia. Int J Cancer 59, 357-362.

34. Karmakar S, Banik NL \& Ray SK (2007) Molecular mechanism of inositol hexaphosphate-mediated apoptosis in human malignant glioblastoma T98G cells. Neurochem Res 32, 2094-2102.

35. Lee M, Wrensch M \& Miike R (1997) Dietary and tobacco risk factors for adult onset glioma in the San Francisco Bay Area (California, USA). Cancer Causes Control 8, 13-24.

36. Blowers L, Preston-Martin S \& Mack WJ (1997) Dietary and other lifestyle factors of women with brain gliomas in Los Angeles County (California, USA). Cancer Causes Control 8, 5-12. 